\title{
On a General Class of Discrete Bivariate DisTRIBUTIONS
}

\author{
DEBASIS Kundu *
}

\begin{abstract}
In this paper we develop a very general class of bivariate discrete distributions. The basic idea is very simple. The marginals are obtained by taking the random geometric sum of a baseline distribution function. The proposed class of distributions is a very flexible class of distributions in the sense the marginals can take variety of shapes. It can be multimodal as well as heavy tailed also. It can be both over dispersed as well as under dispersed. Moreover, the correlation can be of a wide range. We discuss different properties of the proposes class of bivariate distributions. The proposed distribution has some interesting physical interpretations also. Further, we consider two specific base line distributions namely; Poisson and negative binomial distributions for illustrative purposes. Both of them are infinitely divisible. The maximum likelihood estimators of the unknown parameters cannot be obtained in closed form. They can be obtained by solving three and five dimensional non-linear optimizations problems, respectively. To avoid that we propose to use the method of moment estimators and they can be obtained quite conveniently. The analyses of two real data sets have been performed to show the effectiveness of the proposed class of models. Finally, we discuss some open problems and conclude the paper.
\end{abstract}

Key Words And Phrases: Discrete distributions; joint probability mass function; bivariate generating function; infinite divisibility, method of moment estimators.

AMS Subject Classifications: 62F10, 62F03, 62H12.

*Department of Mathematics and Statistics, Indian Institute of Technology Kanpur, Pin 208016, India. E-mail: kundu@iitk.ac.in, Phone no. 91-512-2597141, Fax no. 91-512-2597500. 


\section{INTRODUCTION}

Bivariate lifetime distributions are mainly used to analyze the marginals and also to study the dependence structure of the two marginals. An extensive amount of work has been done to propose different continuous bivariate distributions, analyzing their properties and develop different estimation procedures of the unknown parameters. Some of the early references on bivariate distributions are Gumbel [9], Freund [8], Marshall and Olkin [24], Block and Basu [4] and for some of the recent work one is referred to Balakrishnan and Lai [1], Kundu and Gupta [18], Kundu et al. [17], Lee and Cha [22], Sankaran and Kundu [27] and the references cited therein.

It may be mentioned that that in many practical situations, even if the data are discrete, they are modelled by continuous distributions mainly due to analytical tractability. But, in many practical situations, the discrete data occur quite naturally. Sometimes, it is impossible to measure life length of a device on a continuous scale. In many practical situations lifetimes are recorded on a discrete scale. For example, the on/off switching devices, the number of accidents, to and fro motion of spring devices etc. are purely discrete in nature. Bivariate discrete data also occur quite naturally in practice. For example, the number of goals scored in a football match by two competing teams or the number of insurance claims for two different causes is purely discrete in nature.

An extensive amount of work has been done introducing different bivariate discrete distributions, analyzing their properties and developing different estimation procedures. Special attention has been paid on bivariate geometric distributions and bivariate Poisson distributions, see for example Kocherlakota and Kocherlakota [12], Kocherlakota [13], Basu and Dhar [3], Kumar [14], Kemp [11], Lee and Cha [22, Nekoukhou and Kundu [25], Kundu and Nekoukhou [19] and see the references cited therein. 
Recently, Lee and Cha [23] proposed two classes of discrete bivariate distributions and discussed their properties. Their idea is based on the minimum and maximum of two independent non-identical distributed random variables. The idea is quite simple, and it produces different unimodal shapes of bivariate discrete distributions. Unfortunately, because of the non-identical distributions, the joint probability mass functions (PMFs) or the marginal PMFs may not be in a convenient form. It makes it difficult to compute the estimates of the unknown parameters, and to derive different properties. Moreover, the marginals produced by the method of Lee and Chao [23] cannot have multimodal or heavy tailed property, see also Nekoukhou and Kundu [25] in this respect.

The main aim of this paper is two fold. First of all we develop a very flexible class of discrete bivariate distributions. The proposed discrete bivariate distribution has a very convenient joint probability generating function (PGF), hence the joint PMF can be obtained in a convenient form. The main idea is quite simple. We consider geometric random sum of independent identically distributed (i.i.d.) base line random variables. The idea is not new. It has been used in case of continuous random variables by several authors. For example, Chahkandi and Ganjali [6] used this idea when the base distribution is exponential and Barreto-Souza [2] extended the results in case of gamma distribution. The author [15] considered the case when the base distribution is univariate normal and the results have been recently generalized for multivariate normal base distribution by the author [16]. For some of the related literature, interested readers are referred to Kuzobowski et al. [20] and [21]. Although, the above method seems to be a powerful method and it has been successfully used for several continuous distributions, no attempt has been made in case of discrete distributions. This is an attempt towards that direction.

The main advantage of the proposed method is that it produces a very flexible class of distribution functions. In this case the marginals PMFs can be unimodal or multimodal, 
moreover they can be heavy tailed also. Since, many well known discrete distributions like Poisson, geometric and negative binomial have convolution properties, several interesting properties of the proposed distributions can be established. The proposed model has some interesting physical interpretations also. We derive different properties of the proposed class of distributions in general and discuss in details two special cases. It is observed that the marginals can be both under dispersed as well as over dispersed also, the correlation can be of a wide range, hence it can be used quite conveniently for different data analysis purposes.

Estimation of the unknown parameters is always an important problem in any data analysis. In this case it is observed that the maximum likelihood estimators (MLEs) of the unknown parameters cannot be obtained in closed forms. It involves solving higher dimensional optimization problems. To avoid that we propose to use method of moment estimators (MMEs). It is observed that when the base line distribution is Poisson or negative binomial the MMEs can be obtained quite conveniently. We analyze two real data sets for illustrative purposes.

The rest of the paper is organized as follows. In Section 2, we provide some motivations of the proposed model. The model formulation and some basic properties are discussed in Section 3. In Sections 4 and 5, we discuss two special cases. The analyses of two data sets are presented in Section 6, and finally in Section 7 we conclude the paper.

\section{Motivations}

To motivate our proposed model we will start with an example. First let us consider the traditional construction of the bivariate Poisson distribution. It is based on a trivariate reduction technique and it can be described as follows. Suppose $X_{1}, X_{2}$ and $X_{3}$ are three independent Poisson random variables with mean $\lambda_{1}, \lambda_{2}$ and $\lambda_{3}$, respectively. Consider a 
new bivariate random variable $\left(Y_{1}, Y_{2}\right)$, where

$$
Y_{1}=X_{1}+X_{3} \quad \text { and } \quad Y_{2}=X_{2}+X_{3}
$$

It was originally proposed by Holgate [10], see also Campbell [5] in this respect. The joint PMF of $Y_{1}$ and $Y_{2}$ can be obtained as

$$
P\left(Y_{1}=i, Y_{2}=j\right)=e^{-\left(\lambda_{1}+\lambda_{2}+\lambda_{3}\right)} \sum_{k=0}^{\min \{i, j\}} \frac{\lambda_{1}^{i-k} \lambda_{2}^{j-k} \lambda_{3}^{k}}{(i-k) !(j-k) ! k !} ; \quad i, j \in \mathbb{N}_{0}
$$

where $\mathbb{N}_{0}=\{0,1,2, \ldots\}$. In this case the marginals will follow Poisson distribution. Clearly, this is an advantage of this model. But the marginal PMFs cannot be heavy tailed or have multimodal properties. Due to presence of the summation in the joint PMF computing the MLEs become difficult. Moreover, it may not be very easy to generalize it for other bivariate distributions.

Recently Lee and Cha [23] introduced two very general methods to generate bivariate discrete distribution functions. The methods can be briefly described as follows. Let $X_{1}, X_{2}$ and $X_{3}$ be same as defined before.

LeE-Cha Method 1:

$$
U_{1}=\max \left\{X_{1}, X_{3}\right\} \quad \text { and } \quad U_{2}=\max \left\{X_{1}, X_{3}\right\}
$$

\section{LeE-Cha Method 2:}

$$
V_{1}=\min \left\{X_{1}, X_{3}\right\} \quad \text { and } \quad V_{2}=\min \left\{X_{1}, X_{3}\right\}
$$

In both cases the generated bivariate distributions can be quite flexible. The joint PMF and the marginals PMFs can be of different shapes. Unfortunately, in this case also it has been observed, see Nekoukhou and Kundu [25], that the marginals may not be in a very convenient form. Moreover, in this case also in case of standard discrete distributions like Poisson, geometric or binomial, the marginal PMFs cannot be multimodal or heavy tailed. 
Most of the other bivariate distributions as proposed by Kumar [14] and Piperigou and Papageorgiou [26] have similar deficiencies.

The main aim of this paper is to propose a class of discrete bivariate distributions which has convenient joint PMF and marginal PMFs. The joint PMF and the marginal PMFs should be flexible enough and the marginals PMFs can have heavy tailed and multimodal shapes.

\section{A general Class of Discrete Bivariate Distribu- TIONS}

In this section we develop a general class of discrete bivariate distributions and discuss its different properties. Two special cases will be discussed in the subsequent sections.

Suppose $U_{1}, U_{2}, \ldots$, are independent identically distributed (i.i.d.) random variables with the probability mass function $(\mathrm{PMF}) f_{1}(x)$, for $x \in \mathbb{N}_{0}=\{0,1,2, \ldots\}$. Further, $V_{1}, V_{2}, \ldots$, are i.i.d. random variables with the $\operatorname{PMF} f_{2}(x)$, for $x \in \mathbb{N}_{0}$, and $N$ is a geometric random variable with the PMF

$$
P(N=n)=\theta(1-\theta)^{n-1} ; \quad n \in \mathbb{N}=\{1,2, \ldots\}
$$

for $0<\theta<1$. All the random variables are independently distributed. We define the bivariate random variable $(X, Y)$ as follows

$$
X=\sum_{i=1}^{N} U_{i} \quad \text { and } \quad Y=\sum_{i=1}^{N} V_{i}
$$

The above bivariate random variable $(X, Y)$ has the following physical interpretations.

Accident Model: Suppose $N$ is the number of accidents that took place in a given place during a fixed period of time. Let $U_{i}$ and $V_{i}$ denote the number of deaths and number of 
seriously injured individuals, respectively, due to the $i$-th accident for $i=0,1, \ldots, N$. Then $X$ and $Y$ denote the total number of deaths and total number of seriously injured individuals during that fixed period of time due to accidents in that given place.

Soccer Model: Suppose $N$ denotes the number of soccer games played during a year between Team A and Team B. Suppose $U_{i}$ and $V_{i}$ denote the number of goal scored by Team A and Team B, respectively, at the $i$-th game, for $i=0,1, \ldots, N$. Then $X$ and $Y$ denote the total number goals scored by Team A and Team B, respectively, against each other in that year.

From now on we will call $f_{1}(x)$ and $f_{2}(y)$ as the base line PMFs. First we would like to derive different properties of the bivariate random variable $(X, Y)$ for general base line PMFs. We use the following notations. $F_{1}(x)$ and $F_{2}(x)$ denote the cumulative distribution functions (CDFs) of $f_{1}(x)$ and $f_{2}(x)$, respectively. Moreover, $\phi_{1}(t)$ and $\phi_{2}(s)$ denote the moment generating functions (MGFs) of $f_{1}(x)$ and $f_{2}(x)$, respectively. We define $f_{1}^{(n)}(x)$ and $f_{2}^{(n)}(y)$ as the $n$-fold convolutions of $f_{1}(x)$ and $f_{2}(x)$, respectively, i.e.

$$
f_{1}^{(n)}(x)=P\left(U_{1}+\ldots+U_{n}=x\right) \quad \text { and } \quad f_{2}^{(n)}(y)=P\left(V_{1}+\ldots+V_{n}=y\right)
$$

We further define $F_{1}^{(n)}(x)$ and $F_{2}^{(n)}(y)$ are the CDFs correspond to the PMFs $f_{1}^{(n)}(x)$ and $f_{2}^{(n)}(y)$, respectively. The joint PMF of $X$ and $Y$ for $m=0,1, \ldots$ and $n=0,1, \ldots$, can be obtained as follows:

$$
\begin{aligned}
P(X=m, Y=n) & =P\left(\sum_{i=1}^{N} U_{i}=m, \sum_{j=1}^{N} V_{j}=n\right) \\
& =\sum_{k=1}^{\infty} P\left(\sum_{i=1}^{N} U_{i}=m, \sum_{j=1}^{N} V_{j}=n \mid N=k\right) P(N=k) \\
& =\sum_{k=1}^{\infty} P\left(\sum_{i=1}^{k} U_{i}=m, \sum_{j=1}^{k} V_{j}=n\right) P(N=k) \\
& =p \sum_{k=1}^{\infty}(1-p)^{k-1} f_{1}^{(k)}(m) f_{2}^{(k)}(n) .
\end{aligned}
$$


The joint CDF of $X$ and $Y$ for $m=0,1, \ldots$ and $n=0,1, \ldots$, is

$$
P(X \leq m, Y \leq n)=p \sum_{k=1}^{\infty}(1-p)^{k-1} F_{1}^{(k)}(m) F_{2}^{(k)}(n) .
$$

From the joint PMF of $X$ and $Y$, we immediately obtain the marginals PMFs of $X$ and $Y$ as

$$
\begin{gathered}
P(X=m)=p \sum_{k=1}^{\infty}(1-p)^{k-1} f_{1}^{(k)}(m) \quad \text { and } \quad P(Y=n)=p \sum_{k=1}^{\infty}(1-p)^{k-1} f_{2}^{(k)}(n) . \\
P(X \leq m)=p \sum_{k=1}^{\infty}(1-p)^{k-1} F_{1}^{(k)}(m) \quad \text { and } \quad P(Y \leq n)=p \sum_{k=1}^{\infty}(1-p)^{k-1} F_{2}^{(k)}(n) .
\end{gathered}
$$

Let us assume that $\phi_{1}(t)$ and $\phi_{2}(s)$ exist for $t \in A$ and $s \in B$, where $A, B \subset \mathbb{R}$. Then for $(t, s) \in A \times B$, the joint MGF of $X$ and $Y$ is

$$
\begin{aligned}
\phi_{X, Y}(t, s)=E\left(e^{t X+s Y}\right) & =p \sum_{m=0}^{\infty} \sum_{n=0}^{\infty} \sum_{k=1}^{\infty} e^{t m+s n}(1-p)^{k-1} f_{1}^{(k)}(m) f_{2}^{(k)}(n) \\
& =p \sum_{k=1}^{\infty}(1-p)^{k-1} \sum_{m=0}^{\infty} e^{t m} f_{1}^{(k)}(m) \sum_{n=0}^{\infty} e^{s n} f_{2}^{(k)}(n) \\
& =p \sum_{k=1}^{\infty}(1-p)^{k-1} \phi_{1}^{k}(t) \phi_{2}^{k}(s) \\
& =\frac{p \phi_{1}(t) \phi_{2}(s)}{1-(1-p) \phi_{1}(t) \phi_{2}(s)} .
\end{aligned}
$$

From the joint MGF of $X$ and $Y$, we obtain for $t \in A$ and $s \in B$,

$$
\phi_{X}(t)=\frac{p \phi_{1}(t)}{1-(1-p) \phi_{1}(t)} \quad \text { and } \quad \phi_{Y}(s)=\frac{p \phi_{2}(s)}{1-(1-p) \phi_{2}(s)} .
$$

Moreover, it is immediate from (므) that $X$ and $Y$ are independent if and only if $p=1$. Using the MGFs or otherwise, different moments and product moments can be easily obtained.

$$
\begin{gathered}
E(X)=\frac{E\left(U_{1}\right)}{p}, \quad E(Y)=\frac{E\left(U_{2}\right)}{p} \\
V(X)=\frac{(1-p)\left(E\left(U_{1}\right)\right)^{2}}{p^{2}}+\frac{V\left(U_{1}\right)}{p} \quad V(Y)=\frac{(1-p)\left(E\left(V_{1}\right)\right)^{2}}{p^{2}}+\frac{V\left(V_{1}\right)}{p} \\
\operatorname{Cov}(X, Y)=\frac{1-p}{p^{2}} \quad E\left(U_{1}\right) E\left(V_{1}\right) .
\end{gathered}
$$


Some of the points are quite clear from the above moments expressions. It is clear that as $p \rightarrow 0$, then the mean and variance go to $\infty$. It implies that the marginals become heavy tailed. The correlation between $X$ and $Y$ is always positive and it goes to zero, as $p \rightarrow 1$, and it goes to 1 , as $p \rightarrow 0$. The variance to mean ratio (VMR) for $X$ and $Y$ are

$$
\operatorname{VMR}(X)=\frac{(1-p) E\left(U_{1}\right)}{p}+\operatorname{VMR}\left(U_{1}\right) \quad \text { and } \quad \operatorname{VMR}(Y)=\frac{(1-p) E\left(V_{1}\right)}{p}+\operatorname{VMR}\left(V_{1}\right) .
$$

It is clear that as $p \rightarrow 0$, the marginals will be over dispersed, and for large $p$, the marginals can be under dispersed in the base line distributions are under dispersed. Therefore, it is possible to have both over dispersed and under dispersed marginals for the proposed bivariate distributions. It is also possible to have two opposite behavior of the two marginals. Now we will consider some special cases in the subsequent sections.

\section{Two Special Cases}

\subsection{Bivariate Poisson Geometric}

In this section it is assumed that $U_{i}$ follows $(\sim)$ a Poisson random variable with mean $\lambda_{1}$ (PO $\left.\left(\lambda_{1}\right)\right)$ and $V_{i} \sim \mathrm{PO}\left(\lambda_{2}\right)$. We denote this new distribution as BPG $\left(\lambda_{1}, \lambda_{2}, p\right)$, and the marginals will be denoted by UPG $\left(\lambda_{1}, p\right)$ and UPG $\left(\lambda_{2}, p\right)$, respectively.

The joint PMF of $X$ and $Y$ for $m=0,1, \ldots$ and $n=0,1, \ldots$, can be obtained as follows:

$$
P(X=m, Y=n)=C\left(\lambda_{1}+\lambda_{2}-\ln (1-p), m+n\right) \times \frac{p}{1-p} \times \frac{\lambda_{1}^{m} \lambda_{2}^{n}}{m ! n !} .
$$

Here for $a>0$ and $j=0,1,2, \ldots$,

$$
C(a, j)=\sum_{k=1}^{\infty} k^{j} e^{-a k}
$$

The exact expressions of $C(a, j)$ for different values of $j$ can be obtained recursively, and it is provided in the Appendix A. The marginal PMF of $X$ for $m=0,1,2, \ldots$, and of $Y$ for 
$n=0,1,2, \ldots$ can be obtained as,

$$
\begin{aligned}
P(X=m) & =C\left(\lambda_{1}-\ln (1-p), m\right) \times \frac{p}{1-p} \times \frac{\lambda_{1}^{m}}{m !} \\
P(Y=n) & =C\left(\lambda_{2}-\ln (1-p), n\right) \times \frac{p}{1-p} \times \frac{\lambda_{2}^{n}}{n !}
\end{aligned}
$$

respectively.

$$
P(X=m \mid Y=n)=\frac{C\left(\lambda_{1}+\lambda_{2}-\ln (1-p), m+n\right)}{C\left(\lambda_{1}-\ln (1-p), m\right)} \times \frac{\lambda_{1}^{m}}{m !} .
$$

The joint moment generating function (MGF) of $X$ and $Y$ for $-\infty<s, t<\infty$ is

$$
\phi_{X, Y}(t, s)=E\left(e^{t X+s Y}\right)=\frac{p e^{\lambda_{1}\left(e^{t}-1\right)} e^{\lambda_{2}\left(e^{s}-1\right)}}{1-(1-p) e^{\lambda_{1}\left(e^{t}-1\right)} e^{\lambda_{2}\left(e^{s}-1\right)}} .
$$

Hence the MGF of $X$ and $Y$ for $-\infty<t<\infty$, are

$$
\phi_{X}(t)=E e^{t X}=\frac{p e^{\lambda_{1}\left(e^{t}-1\right)}}{1-(1-p) e^{\lambda_{1}\left(e^{t}-1\right)}} \quad \text { and } \quad \phi_{Y}(t)=E e^{t Y}=\frac{p e^{\lambda_{2}\left(e^{t}-1\right)}}{1-(1-p) e^{\lambda_{2}\left(e^{t}-1\right)}} \quad \text { and }
$$

Different moments and correlation are

$$
\begin{gathered}
E(X)=\frac{\lambda_{1}}{p}, \quad E(Y)=\frac{\lambda_{2}}{p}, \quad V(X)=\frac{\lambda_{1}^{2}}{p^{2}}, \quad V(Y)=\frac{\lambda_{2}^{2}}{p^{2}}, \quad \operatorname{Corr}(X, Y)=(1-p) . \\
\operatorname{Cov}(X, Y)=\frac{1-p}{p^{2}} \quad E\left(U_{1}\right) E\left(V_{1}\right) .
\end{gathered}
$$

The VMR of $X$ and $Y$ are

$$
\operatorname{VMR}(X)=\frac{\lambda_{1}}{p} \quad \text { and } \quad \operatorname{VMR}(Y)=\frac{\lambda_{2}}{p}
$$

Hence, depending on the parameters, the marginals can be over dispersed or under dispersed. We have the following results regarding stochastic ordering of the Poisson geometric distribution. The proofs are quite simple, hence the details are avoided.

Result 1: If $(X, Y) \sim \operatorname{BPG}\left(\lambda_{1}, \lambda_{2}, p\right)$ and $\lambda_{1}>\lambda_{2}$, then $X$ is stochastically larger than $Y$. 
Result 2: If $U \sim \operatorname{UPG}\left(\lambda, p_{1}\right)$ and $V \sim \operatorname{UPG}\left(\lambda, p_{2}\right)$, then for $p_{1}<p_{2}, U$ is stochastically larger than $V$.

TheOrEm 1: If $(X, Y) \sim \operatorname{BPG}\left(\lambda_{1}, \lambda_{2}, p\right)$, then we have the following results.

(a) $X+Y \sim \operatorname{UPG}\left(\lambda_{1}+\lambda_{2}, p\right)$.

(b) $X$ given $X+Y$ follows a binomial distribution, i.e.

$$
P(X=m \mid X+Y=n)=\left(\begin{array}{c}
n \\
m
\end{array}\right)\left(\frac{\lambda_{1}}{\lambda_{1}+\lambda_{2}}\right)^{m}\left(\frac{\lambda_{2}}{\lambda_{1}+\lambda_{2}}\right)^{n-m} ; \quad n=0,1, \ldots, m .
$$

Proof: (a) can be obtained from the joint MGF. (b) can be obtained using (a).

Theorem 2: If $\left\{\left(X_{i}, Y_{i}\right) ; i=1,2, \ldots\right\}$ is a sequence i.i.d. random variables from $\operatorname{BPG}\left(\lambda_{1}, \lambda_{2}, p\right), M$ is a geometric random variable with parameter $\delta$, for $0<\delta<1$, and for $i=1, \ldots$, it is independent of $\left(X_{i}, Y_{i}\right)$, then

$$
\left(\sum_{i=1}^{M} X_{i}, \sum_{i=1}^{M} Y_{i}\right) \sim \operatorname{BPG}\left(\lambda_{1}, \lambda_{2}, p \delta\right) .
$$

PRoof: The proof mainly follows from the joint MGF.

Theorem 3: If $(X, Y) \sim \operatorname{BPG}\left(\lambda_{1}, \lambda_{2}, p\right)$, then $(X, Y)$ is infinitely divisible.

Proof: Note that we need to prove that for any positive integer $n$, there exists i.i.d. random variables $\left\{\left(W_{1 i}^{(n)}, W_{2 i}^{(n)}\right) ; i=1,2, \ldots, n\right\}$, such that $\left(W_{1 i}^{(n)}, W_{2 i}^{(n)}\right)$ follows bivariate Poisson geometric random variables, and

$$
(X, Y) \stackrel{d}{=}\left(\sum_{i=1}^{n} W_{1 i}^{(n)}, \sum_{i=1}^{n} W_{2 i}^{(n)}\right) .
$$

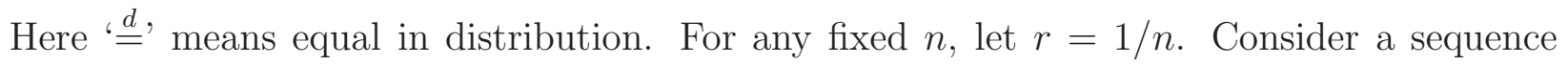
of random i.i.d. random variables $Y_{1}, Y_{2}, \ldots$, such that $Y_{i} \sim \operatorname{PO}\left(r \lambda_{1}\right)$. Similarly, consider another sequence of i.i.d. random variables $Z_{1}, Z_{2}, \ldots$, such that $Z_{i} \sim \mathrm{PO}\left(r \lambda_{2}\right)$. Suppose $T$ 
is a negative binomial distribution $\mathrm{NB}(r, p)$, with the following $\mathrm{PMF}$

$$
P(T=k)=\frac{\Gamma(k+r)}{k ! \Gamma(r)} p^{r}(1-p)^{k} ; \quad k=0,1,2, \ldots
$$

All the above random variables, namely $Y_{i}$ 's $Z_{i}$ 's and $T$ are independently distributed. Consider the following bivariate random variable

$$
\left(W_{11}^{(n)}, W_{21}^{(n)}\right) \stackrel{d}{=}\left(\sum_{i=1}^{1+n T} Y_{i}, \sum_{i=1}^{1+n T} Z_{i}\right)
$$

The joint MGF of $\left(W_{11}^{(n)}, W_{21}^{(n)}\right)$ can be obtained as

$$
\begin{aligned}
\phi_{W_{11}^{(n)}, W_{12}^{(n)}}(t, s) & =E\left(e^{t W_{11}^{(n)}+s W_{21}^{(n)}}\right)=E_{T}\left(E\left(e^{t W_{11}^{(n)}+s W_{21}^{(n)}} \mid T\right)\right) \\
& =E_{T}\left(e^{r \lambda_{1}\left(e^{t}-1\right)} e^{r \lambda_{2}\left(e^{2}-1\right)(1+n T)}\right) \\
& =e^{r \lambda_{1}\left(e^{t}-1\right)} e^{r \lambda_{2}\left(e^{2}-1\right)}\left(\frac{p}{1-(1-p) e^{\lambda_{1}\left(e^{t}-1\right)} e^{\lambda_{2}\left(e^{2}-1\right)}}\right)^{r} \\
& =\left(\frac{p e^{\lambda_{1}\left(e^{t}-1\right)} e^{\lambda_{2}\left(e^{2}-1\right)}}{1-(1-p) e^{\lambda_{1}\left(e^{t}-1\right)} e^{\lambda_{2}\left(e^{2}-1\right)}}\right)^{r} .
\end{aligned}
$$

Hence, the result is obtained.

The following result shows that the bivariate Poisson geometric distribution has an interesting decomposition. It might have some independent interest also.

Theorem 4: Let us assume that $(X, Y) \sim \operatorname{BPG}\left(\lambda_{1}, \lambda_{2}, p\right)$. Suppose $Q \sim \operatorname{PO}(\lambda)$, where $\lambda=-\ln p$, and it is independent of $Z_{i}$ 's, where $\left\{Z_{i} ; i=1,2, \ldots\right\}$ is a sequence of i.i.d. random variables having logarithmic distribution with the probability mass function

$$
P\left(Z_{1}=k\right)=\frac{(1-p)^{k}}{\lambda k} ; \quad k=1,2, \ldots
$$

Moreover, $\left\{W_{1 i} ; i=1,2, \ldots\right\}$ is a sequence of i.i.d. random variables such that $W_{1 i} \mid Z_{i} \sim$ $\mathrm{PO}\left(\lambda_{1} Z_{i}\right)$, similarly, $\left\{W_{2 i} ; i=1,2, \ldots\right\}$ is a sequence of i.i.d. random variables such that $W_{2 i} \mid Z_{i} \sim \mathrm{PO}\left(\lambda_{2} Z_{i}\right)$, and conditionally they are independently distributed. $R$ and $S$ are two 
independent random variables and they are independent of all the previous random variables, such that $R \sim \mathrm{PO}\left(\lambda_{1}\right), S \sim \mathrm{PO}\left(\lambda_{2}\right)$. They we have the following decomposition of $(X, Y)$

$$
(X, Y) \stackrel{d}{=}\left(R+\sum_{i=1}^{Q} W_{1 i}, S+\sum_{i=1}^{Q} W_{2 i}\right) .
$$

Proof: First observe that the probability generating function of $Q$ and $Z_{1}$ are

$$
E\left(t^{Q}\right)=e^{\lambda(t-1)}, \quad t \in \mathbb{R} \quad \text { and } \quad E\left(t^{Z_{1}}\right)=\frac{\ln (1-(1-p) t)}{\ln p}, \quad t<(1-p)^{-1}
$$

The joint MGF of the right hand side of (10) can be written as

$$
\begin{aligned}
\phi(u, v) & =E\left(e^{u\left(R+\sum_{i=1}^{Q} W_{1 i}\right)+v\left(S+\sum_{i=1}^{Q} W_{2 i}\right)}\right) \\
& =e^{\lambda_{1}\left(e^{u}-1\right)} e^{\lambda_{2}\left(e^{v}-1\right)} E\left(e^{u \sum_{i=1}^{Q} W_{1 i}+v \sum_{i=1}^{Q} W_{2 i}}\right) \\
& =e^{\lambda_{1}\left(e^{u}-1\right)} e^{\lambda_{2}\left(e^{v}-1\right)} E_{Q}\left[E\left(e^{u \sum_{i=1}^{Q} W_{1 i}+v \sum_{i=1}^{Q} W_{2 i}} \mid Q\right)\right] \\
& =e^{\lambda_{1}\left(e^{u}-1\right)} e^{\lambda_{2}\left(e^{v}-1\right)} E_{Q} E\left[E\left(e^{u \sum_{i=1}^{Q} W_{1 i}} \mid Q, Z_{1}, Z_{2}, \ldots\right) E\left(e^{v \sum_{i=1}^{Q} W_{2 i}} \mid Q, Z_{1}, Z_{2}, \ldots\right)\right] \\
& =e^{\lambda_{1}\left(e^{u}-1\right)} e^{\lambda_{2}\left(e^{v}-1\right)} E_{Q} E\left[e^{\lambda_{1}\left(e^{u t}-1\right) \sum_{i=1}^{Q} Z_{i}} e^{\lambda_{2}\left(e^{v s}-1\right) \sum_{i=1}^{Q} Z_{i}}\right] \\
& =e^{\lambda_{1}\left(e^{u}-1\right)} e^{\lambda_{2}\left(e^{v}-1\right)} E_{Q}\left[E\left[e^{\left(\lambda_{1}\left(e^{u t}-1\right)+\lambda_{2}\left(e^{v s}-1\right)\right) Z_{1}}\right]\right]^{Q} \\
& =e^{\lambda_{1}\left(e^{u}-1\right)} e^{\lambda_{2}\left(e^{v}-1\right)} E_{Q}\left[\frac{\ln \left(1-(1-p) e^{\left(\lambda_{1}\left(e^{u t}-1\right)+\lambda_{2}\left(e^{v s}-1\right)\right)}\right)}{\ln p}\right. \\
& =e^{\lambda_{1}\left(e^{u}-1\right)} e^{\lambda_{2}\left(e^{v}-1\right)} e^{\ln p-\ln \left(1-(1-p) e^{\left(\lambda_{1}\left(e^{u t}-1\right)+\lambda_{2}\left(e^{v s}-1\right)\right)}\right)} \\
& =\frac{p e^{\lambda_{1}\left(e^{u}-1\right)} e^{\lambda_{2}\left(e^{v}-1\right)}}{1-(1-p) e^{\lambda_{1}\left(e^{u}-1\right)} e^{\lambda_{2}\left(e^{v}-1\right)}} .
\end{aligned}
$$

\subsection{Bivariate Negative Binomial Geometric}

In this section we consider another special case when $U_{1} \sim \mathrm{NB}\left(r_{1}, \theta_{1}\right)$ and $V_{1} \sim \mathrm{NB}\left(r_{2}, \theta_{2}\right)$,

where $r_{1}>0, r_{2}>0,0<\theta_{1}, \theta_{2}<1$. Here $\mathrm{NB}(r, \theta)$ means a negative binomial distribution with the PMF

$$
P(X=k)=\frac{\Gamma(k+r)}{k ! \Gamma(r)} \theta^{k}(1-\theta)^{r} ; \quad k=0,1,2, \ldots
$$


In this case we denote this bivariate distribution as $\operatorname{BNBG}\left(r_{1}, \theta_{1}, r_{2}, \theta_{2}, p\right)$, and the marginals will be denoted by $\operatorname{UNBG}\left(r_{1}, \theta_{1}, p\right)$ and $\operatorname{UNBG}\left(r_{2}, \theta_{2}, p\right)$, respectively. The joint PMF of $X$ and $Y$ for $m=0,1, \ldots$ and $n=0,1, \ldots$, can be written as

$$
P(X=m, Y=n)=D\left(r_{1}, r_{2}, \theta_{1}, \theta_{2}, m, n, p\right) \theta_{1}^{m} \theta_{2}^{n} \frac{p}{1-p}
$$

where

$$
D\left(r_{1}, r_{2}, \theta_{1}, \theta_{2}, m, n, p\right)=\sum_{k=1}^{\infty} \frac{\Gamma\left(m+k r_{1}\right)}{m ! \Gamma\left(k r_{1}\right)} \times \frac{\Gamma\left(n+k r_{2}\right)}{n ! \Gamma\left(k r_{2}\right)}(1-p)^{k}\left(1-\theta_{1}\right)^{k r_{1}}\left(1-\theta_{2}\right)^{k r_{2}}
$$

The marginal PMFs of $X$ and $Y$ can be obtained as

$$
\begin{aligned}
P(X=m) & =D_{1}\left(r_{1}, \theta_{1}, m, p\right) \theta_{1}^{m} \frac{p}{1-p} \\
P(Y=n) & =D_{1}\left(r_{2}, \theta_{2}, n, p\right) \theta_{2}^{m} \frac{p}{1-p}
\end{aligned}
$$

where

$$
D_{1}(r, \theta, m, p)=\sum_{k=1}^{\infty} \frac{\Gamma(m+k r)}{m ! \Gamma(k r)}(1-\theta)^{k r}(1-p)^{k} .
$$

The joint MGF of $X$ and $Y$ for $-\infty<s, t<\infty$, is

$$
\phi_{X, Y}(t, s)=E\left(e^{t X+s Y}\right)=\frac{p\left(1-\theta_{1}\right)^{r_{1}}\left(1-\theta_{2}\right)^{r_{2}}}{\left(1-\theta_{1} e^{t}\right)^{r_{1}}\left(1-\theta_{2} e^{s}\right)^{r_{2}}-(1-p)\left(1-\theta_{1}\right)^{r_{1}}\left(1-\theta_{2}\right)^{r_{2}}} .
$$

The marginal MGFs of $X$ and $Y$ can be obtained as

$$
\phi_{X}(t)=\frac{p\left(1-\theta_{1}\right)^{r_{1}}}{\left(1-\theta_{1} e^{t}\right)^{r_{1}}-(1-p)\left(1-\theta_{1}\right)^{r_{1}}}, \quad \phi_{Y}(s)=\frac{p\left(1-\theta_{2}\right)^{r_{2}}}{\left(1-\theta_{2} e^{s}\right)^{r_{2}}-(1-p)\left(1-\theta_{2}\right)^{r_{2}}} .
$$

Different moments and product moments of the BNBG distribution can be easily obtained and they are as follows.

$$
\begin{gathered}
E(X)=\frac{\theta_{1} r_{1}}{p\left(1-\theta_{1}\right)}, \quad E(Y)=\frac{\theta_{2} r_{2}}{p\left(1-\theta_{2}\right)} \\
V(X)=\frac{(1-p) \theta_{1}^{2} r_{1}^{2}}{p^{2}\left(1-\theta_{1}\right)^{2}}+\frac{\theta_{1} r_{1}}{p\left(1-\theta_{1}\right)^{2}} \quad V(Y)=\frac{(1-p) \theta_{2}^{2} r_{2}^{2}}{p^{2}\left(1-\theta_{2}\right)^{2}}+\frac{\theta_{2} r_{2}}{p\left(1-\theta_{2}\right)^{2}} \\
\operatorname{Cov}(X, Y)=\frac{1-p}{p^{2}} \quad \frac{\theta_{1} r_{1} \theta_{2} r_{2}}{\left(1-\theta_{1}\right)\left(1-\theta_{2}\right)} .
\end{gathered}
$$


Theorem 5: If $(X, Y) \sim \operatorname{BNBG}\left(r_{1}, \theta, r_{2}, \theta, p\right)$, then $X+Y \sim \operatorname{UNBG}\left(r_{1}+r_{2}, \theta, p\right)$.

PRoOF: It can be obtained from the joint MGF.

TheOrem 6: If $\left\{\left(X_{i}, Y_{i}\right) ; i=1,2, \ldots\right\}$ is a sequence i.i.d. random variables from $\operatorname{BNBG}\left(r_{1}, \theta_{1}, r_{2}, \theta_{2}, p\right)$, and $M$ is a geometric random variable with parameter $\delta$, for $0<\delta<1$, then

$$
\left(\sum_{i=1}^{M} X_{i}, \sum_{i=1}^{M} Y_{i}\right) \sim \operatorname{BNBG}\left(r_{1}, \theta_{1}, r_{2}, \theta_{2}, p \delta\right) .
$$

Proof: The proof mainly follows from the joint MGF.

Theorem 7: If $(X, Y) \sim \operatorname{BNBG}\left(r_{1}, \theta_{1}, r_{2}, \theta_{2}, p\right)$, then $(X, Y)$ is infinitely divisible.

Proof: The proof can be obtained along the same line of proof as of Theorem 3, the details are avoided.

Theorem 8: Let us assume that $(X, Y) \sim \operatorname{BNBG}\left(r_{1}, \theta_{1}, r_{2}, \theta_{2}, p\right)$. Suppose $Q \sim \operatorname{PO}(\lambda)$, where $\lambda=-\ln p$, and it is independent of $Z_{i}$ 's, where $\left\{Z_{i} ; i=1,2, \ldots\right\}$ is a sequence of i.i.d. random variables having logarithmic distribution with probability mass function as defined (9). Moreover, $\left\{W_{1 i} ; i=1,2, \ldots\right\}$ is a sequence of i.i.d. random variables such that $W_{1 i} \mid Z_{i} \sim \operatorname{NB}\left(r_{1} Z_{i}, \theta_{1}\right)$, similarly, $\left\{W_{2 i} ; i=1,2, \ldots\right\}$ is a sequence of i.i.d. random variables such that $W_{2 i} \mid Z_{i} \sim \mathrm{NB}\left(r_{2} Z_{i}, \theta_{2}\right)$, and conditionally they are independently distributed. $R$ and $S$ are two independent random variables and they are independent of all the previous random variables, such that $R \sim \mathrm{NB}\left(r_{1}, \theta_{1}\right), S \sim \mathrm{NB}\left(r_{2}, \theta_{2}\right)$. They we have the following decomposition of $(X, Y)$

$$
(X, Y) \stackrel{d}{=}\left(R+\sum_{i=1}^{Q} W_{1 i}, S+\sum_{i=1}^{Q} W_{2 i}\right) .
$$

Proof: The proof can be obtained similarly as the proof of Theorem 4. 


\section{Data Analysis}

\subsection{Italian Football Data:}

In this section we present the analysis of two data sets for illustrative purposes. The first data set represents the Italian Series A football match score data between 'ACF Firontina' $(X)$ and 'Juventus' $(Y)$ during 1990 to 2005. The data set is presented in Table1, We would like to use both BPG and BNBG to analyze this data set. We have used the MMEs of the unknown parameters for both the models. The sample means, variances and correlation are presented below.

$$
\mu_{x}=1.3846, \quad \sigma_{x}^{2}=1.7751, \quad \mu_{y}=1.6923, \quad \sigma_{y}^{2}=2.2130, \quad r_{x, y}=0.1179
$$

Based on the BPG model it can be easily seen that the MMEs of the unknown parameters and the associated bootstrap standard errors (reported within brackets) are

$$
\begin{aligned}
\widetilde{p} & =1-r_{x, y}=0.8821(\mp 0.0211), \\
\widetilde{\lambda}_{1} & =\mu_{x}\left(1-r_{x, y}\right)=1.2214(\mp 0.1154) \\
\widetilde{\lambda}_{2} & =\mu_{y}\left(1-r_{x, y}\right)=1.4928(\mp 0.1987) .
\end{aligned}
$$

Based on the BNBG model it can be easily seen that the MMEs of the unknown parameters and the associated bootstrap standard errors (reported within brackets) are

$$
\begin{aligned}
\widetilde{p} & =1-\frac{r_{x, y} \sigma_{x} \sigma_{y}}{\mu_{x} \mu_{y}}=0.9003(\mp 0.0114) \\
\widetilde{\theta}_{1} & =1-\mu_{x} /\left(\sigma_{x}^{2}-(1-\widetilde{p}) \mu_{x}^{2}\right)=0.1259(\mp 0.0013) \\
\widetilde{\theta}_{2} & =1-\mu_{y} /\left(\sigma_{y}^{2}-(1-\widetilde{p}) \mu_{y}^{2}\right)=0.1221(\mp 0.0019) \\
\widetilde{r}_{1} & =\mu_{x} \widetilde{p}\left(1-\widetilde{\theta}_{1}\right) / \widetilde{\theta}_{1}=8.6546(\mp 0.7975) \\
\widetilde{r}_{2} & =\mu_{y} \widetilde{p}\left(1-\widetilde{\theta}_{2}\right) / \widetilde{\theta}_{2}=10.9545(\mp 1.1125)
\end{aligned}
$$




\begin{tabular}{|l|c|c|c||c|c|}
\hline Obs. & $\begin{array}{c}\text { ACF } \\
\text { Firontina } \\
(X)\end{array}$ & $\begin{array}{c}\text { Juventus } \\
(Y)\end{array}$ & Obs. & $\begin{array}{c}\text { ACF } \\
\text { Firontina } \\
(X)\end{array}$ & $\begin{array}{c}\text { Juventus } \\
(Y)\end{array}$ \\
\hline \hline 1 & 1 & 2 & 14 & 4 & 1 \\
2 & 0 & 0 & 15 & 4 & 4 \\
3 & 0 & 0 & 16 & 1 & 3 \\
4 & 2 & 2 & 17 & 1 & 3 \\
5 & 4 & 3 & 18 & 0 & 0 \\
6 & 0 & 1 & 19 & 1 & 0 \\
7 & 1 & 0 & 20 & 0 & 2 \\
8 & 3 & 2 & 21 & 3 & 0 \\
9 & 1 & 3 & 22 & 3 & 0 \\
10 & 2 & 0 & 23 & 1 & 2 \\
11 & 1 & 2 & 24 & 1 & 4 \\
12 & 2 & 3 & 25 & 0 & 2 \\
13 & 0 & 0 & 26 & 0 & 5 \\
\hline
\end{tabular}

Table 1: UEFA Champion's League data

\subsection{Seizure Data}

This data set represents weekly seizure data of 30 patients reported in Davis [7]. Here the first column $(X)$ and the second column $(Y)$ represent the number of seizures observed on each patient in the first week and second week, respectively, after admission to the hospital. The data set is presented in Table 2. In this case also we would like to use both BPG and BNBG to analyze this data set. The sample means, variances and correlation are presented below.

$$
\mu_{x}=1.5667, \quad \sigma_{x}^{2}=1.7789, \quad \mu_{y}=0.9667, \quad \sigma_{y}^{2}=1.4322, \quad r_{x, y}=0.0327 .
$$

Based on the BPG model it can be easily seen that the MMEs of the unknown parameters and the associated bootstrap standard errors (reported within brackets) are

$$
\begin{aligned}
\widetilde{p} & =1-r_{x, y}=0.9673(\mp 0.0132), \\
\widetilde{\lambda}_{1} & =\mu_{x}\left(1-r_{x, y}\right)=1.5155(\mp 0.1765)
\end{aligned}
$$




\begin{tabular}{|l|c|c|c||c|c|}
\hline Obs. & Week-1 & Week-2 & Obs. & Week-1 & Week-2 \\
& $(X)$ & $(Y)$ & & $(X)$ & $(Y)$ \\
\hline \hline 1 & 5 & 0 & 16 & 1 & 0 \\
2 & 1 & 2 & 17 & 1 & 3 \\
3 & 1 & 4 & 18 & 0 & 2 \\
4 & 3 & 2 & 19 & 1 & 1 \\
5 & 3 & 1 & 20 & 2 & 1 \\
6 & 0 & 0 & 21 & 0 & 0 \\
7 & 1 & 0 & 22 & 2 & 1 \\
8 & 4 & 0 & 23 & 1 & 4 \\
9 & 0 & 0 & 24 & 1 & 0 \\
10 & 3 & 2 & 25 & 0 & 0 \\
11 & 3 & 0 & 26 & 2 & 2 \\
12 & 3 & 2 & 27 & 0 & 0 \\
13 & 1 & 0 & 28 & 1 & 1 \\
14 & 3 & 2 & 29 & 6 & 0 \\
15 & 0 & 0 & 30 & 3 & 0 \\
\hline
\end{tabular}

Table 2: Weekly seizure data on 30 patients

$$
\widetilde{\lambda}_{2}=\mu_{y}\left(1-r_{x, y}\right)=0.9351(\mp 0.0967) .
$$

Based on the BNBG model it can be easily seen that the MMEs of the unknown parameters and the associated bootstrap standard errors (reported within brackets) are

$$
\begin{aligned}
\widetilde{p} & =1-\frac{r_{x, y} \sigma_{x} \sigma_{y}}{\mu_{x} \mu_{y}}=0.9655(\mp 0.0178), \\
\widetilde{\theta}_{1} & =1-\mu_{x} /\left(\sigma_{x}^{2}-(1-\widetilde{p}) \mu_{x}^{2}\right)=0.0753(\mp 0.0004) \\
\widetilde{\theta}_{2} & =1-\mu_{y} /\left(\sigma_{y}^{2}-(1-\widetilde{p}) \mu_{y}^{2}\right)=0.3094(\mp 0.0065) \\
\widetilde{r}_{1} & =\mu_{x} \widetilde{p}\left(1-\widetilde{\theta}_{1}\right) / \widetilde{\theta}_{1}=18.5756(\mp 1.2341) \\
\widetilde{r}_{2} & =\mu_{y} \widetilde{p}\left(1-\widetilde{\theta}_{2}\right) / \widetilde{\theta}_{2}=2.0833(\mp 0.0653)
\end{aligned}
$$




\section{Conclusions}

In this section we have proposed a very general bivariate discrete distributions which is a very flexible class of distributions. Due to presence of an extra parameter, the proposed class of distributions is more flexible than the base distributuion functions. It can take variety of shapes, and it can be both over dispersed as well as under dispersed depending on the parameters. We have discussed several properties of the proposed class of distributions and consider two special cases, namely BPG and BNBG distributions. It is observed that both BPG and BNBG are infinitely divisible and they have some interesting physical interpretations also. We have proposed to use the MMEs to estimate the unknown parameters and they can be obtained very conveniently and analyze two data sets for illustrative purposes.

Although, in this paper we have proposed the bivariate class of distributions, the method can be applied even for multivariate case also. Moreover, in this paper we have not developed any inference procedure based on the maximum likelihood approach. It involves solving higher dimensional optmization problems. Efficient algorithm is needed to compute the maximum likelihood estimators in this case. More work is needed along that direction.

\section{ApPENDiX: ExACT Expressions of $C(a, j)$}

Note that for

$$
C(a, 0)=\sum_{k=1}^{\infty} e^{-a k}=\frac{e^{-a}}{1-e^{-a}}=\frac{1}{e^{a}-1} .
$$

To compute $C(a, 1)$, first observe that

$$
C(a, 1)=\sum_{k=1}^{\infty} e^{-a k} k=\frac{1}{e^{a}}+\frac{2}{e^{2 a}}+\frac{3}{e^{3 a}}+\cdots
$$

and

$$
e^{a} C(a, 1)=1+\frac{2}{e^{a}}+\frac{3}{e^{2 a}}+\frac{4}{e^{3 a}}+\cdots
$$


Hence

$$
\left(e^{a}-1\right) C(a, 1)=1+\frac{1}{e^{a}}+\frac{1}{e^{2 a}}+\frac{1}{e^{3 a}}+\cdots=\frac{e^{a}}{e^{a}-1} .
$$

Therefore

$$
C(a, 1)=\frac{e^{a}}{\left(e^{a}-1\right)^{2}} .
$$

Now to compute $C(a, 2)$, note that

$$
C(a, 2)=\sum_{k=0}^{\infty} e^{-a k} k^{2}=\sum_{k=0}^{\infty} e^{-a k} k(k-1)+\sum_{k=0}^{\infty} e^{-a k} k .
$$

If we denote $S=\sum_{k=0}^{\infty} e^{-a k} k(k-1)$, then

$$
S=\frac{2 \cdot 1}{e^{2 a}}+\frac{3 \cdot 2}{e^{3 a}}+\frac{4 \cdot 3}{e^{4 a}}+\cdots
$$

and

$$
e^{a} S=\frac{2 \cdot 1}{e^{a}}+\frac{3 \cdot 2}{e^{2 a}}+\frac{4 \cdot 3}{e^{3 a}}+\cdots
$$

Hence

$$
S\left(e^{a}-1\right)=\frac{2 \cdot 1}{e^{a}}+\frac{2 \cdot 2}{e^{2 a}}+\frac{2 \cdot 3}{e^{3 a}}+\cdots=2 \sum_{k=1}^{\infty} k e^{-a k}=\frac{2 e^{a}}{\left(e^{a}-1\right)^{2}} .
$$

Therefore,

$$
C(a, 2)=\frac{2 e^{a}}{\left(e^{a}-1\right)^{3}}+\frac{e^{a}}{\left(e^{a}-1\right)^{2}}=\frac{e^{2 a}+e^{a}}{\left(e^{a}-1\right)^{3}} .
$$

We will use the following notations:

$$
S_{0}(a)=\sum_{k=0}^{\infty} k e^{-k a}, \quad S_{1}(a)=\sum_{k=0}^{\infty} k(k-1) e^{-k a}, \ldots, S_{m}(a)=\sum_{k=0}^{\infty} k(k-1) \cdots(k-m) e^{-k a} .
$$

Then using the fact

$$
e^{a} S_{m}(a)=\sum_{k=0}^{\infty} k(k-1) \cdots(k-m) e^{-(k-1) a} .
$$

We can easily obtain the following relation

$$
S_{m}(a)\left(e^{a}-1\right)=(m+1) S_{m-1}(a) .
$$


Further note that if we denote

$k^{m}=C_{0 m} k(k-1) \cdots(k-m+1)+C_{1 m} k(k-1) \cdots(k-m+1)+\cdots+C_{m-2, m} k(k-1)+C_{m-1, m} k$,

then $C_{0 m}, C_{1 m} \cdots, C_{m-1, m}$ can be obtained recursively from the following set of linear equations. $C_{0 m}=1$ and

$$
\begin{aligned}
-C_{0 m} \sum_{1 \leq i_{1} \leq m-1} i_{1}+C_{1 m} & =0 \\
C_{0 m} \sum_{1 \leq i_{1}<i_{2} \leq m-1} i_{1} i_{2}-C_{1 m} \sum_{1 \leq i_{1} \leq m-2} i_{1}+C_{2 m} & =0 \\
\sum_{0 m} i_{1} i_{2} i_{3}+C_{1 m} \sum_{1 \leq i_{1}<i_{2} \leq m-2} i_{1} i_{2}-C_{2 m} \sum_{1 \leq i_{1} \leq m-3} i_{1}+C_{3 m} \leq m-1 & =0 \\
(-1)^{m-1} C_{0 m} \prod_{i=1}^{m-1} i(-1)^{m-2} C_{1 m} \prod_{i=1}^{m-2} i(-1)^{m-3} C_{2 m} \prod_{i=1}^{m-3} i+\cdots-C_{m-2, m}+C_{m-1, m} & =0 .
\end{aligned}
$$

If we use the following notations for $n<m$;

$$
a_{n m}=\sum_{1 \leq i_{1}<i_{2}<\ldots<i_{n} \leq m} i_{1} i_{2} \cdots i_{n}
$$

$a_{m m}=\prod_{i=1}^{m} i$, then clearly

$$
a_{n, m+1}=a_{n, m}+(m+1) a_{n-1, m},
$$

and we obtain

$$
\begin{aligned}
C_{1 m} & =a_{1, m-1} \\
C_{2 m} & =C_{1 m} a_{1, m-2}-a_{2, m-1} \\
C_{3 m} & =C_{2 m} a_{1, m-3}-C_{1 m} a_{2, m-2}+a_{3, m-1} \\
\vdots & =\vdots \\
C_{m-1, m} & =C_{m-2, m} a_{11}-C_{m-3, m} a_{2,2}+\ldots(-1)^{m-2} a_{m-1, m-1} .
\end{aligned}
$$

Since we have

$$
C(a, m)=C_{0 m} S_{m-1}(a)+C_{1 m} S_{m-2}(a)+\ldots+C_{m-1, m} S_{0}(a),
$$


we can obtain recursively $C(a, m+1)$ from $C(a, m)$.

\section{References}

[1] Balakrishnan, N. and Lai, C. (2009), Continuous bivariate distributions, 2nd edition, Springer, New York.

[2] Barreto-Souza, W. (2012), "Bivariate gamma-geometric law and its induced Levy process", Journal of Multivariate Analysis, vol. 109, 130 - 145

[3] Basu, A.P. and Dhar, S.K. (1995), "Bivariate geometric distribution", Journal of Applied Statistical Sciences, vol. 2, $33-34$.

[4] Block, H.W. and Basu, A.P. (1974), "A continuous bivariate exponential distribution", Journal of the American Statistical Association, vol. 69, 1031 - 1037.

[5] Campbell, J.T. (1934), "The Poisson correlation function", Proceedings of the Edinburgh Mathematical Society, Series 2, vol. 4, 18 - 26.

[6] Chahkandi, M. and Ganjali, M. (2009), "On some lifetime distributions with decreasing failure rate", Computational Statistics and Data Analysis, vol. 53, 4433 4440.

[7] Davis, C.S. (2002), Statistical methods for the analysis of repeated measurements, Springer-Verlag, New York.

[8] Freund, J.E. (1961), "A bivariate extension of the exponential distribution", Journal of the American Statistical Association, vol. 56, 971 - 977.

[9] Gumbel, E.J. (1960), "Bivariate exponential distribution", Journal of the American Statistical Association, vol. 55, 698 - 707. 
[10] Holgate, B. (1964), "Estimation for the bivariate Poisson distribution", Biometrika, vol. $51,241-245$.

[11] Kemp, A.W. (2013), "New discrete Appell and Humbert distributions with relevance to bivariate accident data", Journal of Multivariate Analysis, vol. 113, 2-6.

[12] Kocherlakota, S. and Kocherlakota, K. (1992), Bivariate discrete distributions, Marcel and Dekker, New York.

[13] Kocherlakota, S. (1995), "Discrete bivariate weighted distributions under multiplicative weight function", Communications in Statistics - Theory and Methods, vol. $24,533-551$.

[14] Kumar, C.S. (2008) "A unified approach to bivariate discrete data", Metrika, vol. $67,113-123$.

[15] Kundu, D. (2014), "Geometric skewed normal distribution", Sankhya, Ser. B., vol. $76,167-189$.

[16] Kundu, D. (2017), "Multivariate geometric skew normal distribution", Statistics, vol. $51,1377-1397$.

[17] Kundu, D., Balakrishnan, N. and Jamalizadeh, A. (2010), "Bivariate BirnbaumSaunders distribution and associated inference", Journal of Multivariate Analysis, vol. $101,113-125$.

[18] Kundu, D. and Gupta, R.D. (2009), "Bivariate generalized exponential distribution", Journal of Multivariate Analysis, vol. 100, 581 - 593, 2009.

[19] Kundu, D. and Nekoukhou, V. (2018), "Univariate and bivariate geometric discrete generalized exponential distributions", Journal of Statistical Theory and Practice, DOI: $10.1080 / 15598608.2018 .1441082$. 
[20] Kuzobowski, T.J., Panorska, A.K., Podgorski, K. (2008), "A bivariate Levy process with negative binomial and gamma marginals", Journal of Multivariate Analysis, vol. 199, $1418-1437$.

[21] Kuzobowski, T.J., Panorska, A.K., Qeadan, F. (2011), "A new multivariate model involving geometric sums and maxima of exponentials", Journal of Statistical Planning and Inference, vol. 141, 2353 - 2367.

[22] Lee, H. and Cha, J.H. (2014), "On construction of general class of bivariate distributions", Journal of Multivariate Analysis, vol. 127, 151 - 159.

[23] Lee, H. and Cha, J.H. (2015), "On two general classes of discrete bivariate distributions", American Statistician, vol. 69, 221 - 230.

[24] Marshall, A.W. and Olkin, I. (1967), "A multivariate exponential distribution", Journal of the American Statistical Association, vol. 62, 30 - 44.

[25] Nekoukhou, V. and Kundu, D. (2017), "Bivariate discrete generalized exponential distribution", Statistics, vol. 51, $1143-1158$.

[26] Piperigou, V.E. and Papageorgiou, H. (2003), "On bivariate discrete distributions: a unified treatment", Metrika, vol. 58, 221 - 233.

[27] Sankaran, P. G. and Kundu, D. (2014), "On a bivariate Pareto model", Statistics, vol. $48,241-255$. 


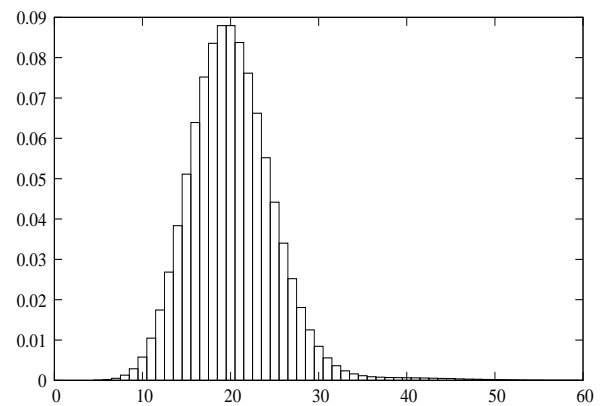

Figure 1: The PMF of a univariate GDGE distribution when $\alpha=1.5, \theta=0.5, p=e^{-1.0}$.

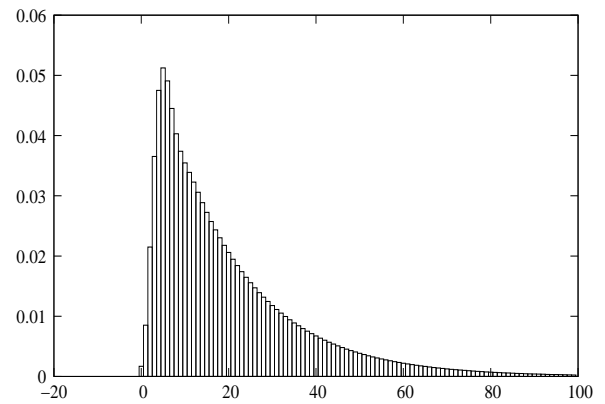

Figure 2: The PMF of a univariate GDGE distribution when $\alpha=1.5, \theta=0.01, p=e^{-1.0}$.

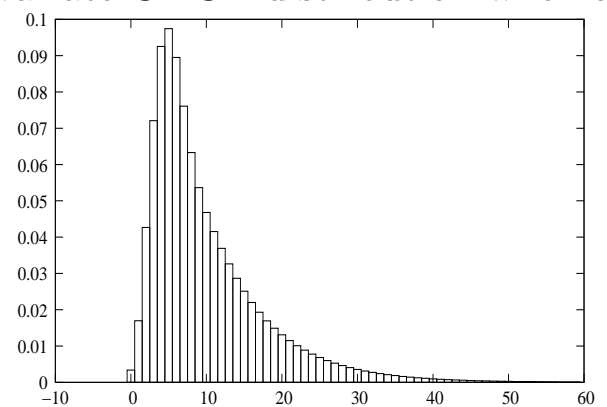

Figure 3: The PMF of a univariate GDGE distribution when $\alpha=1.5, \theta=0.01, p=e^{-0.1}$.

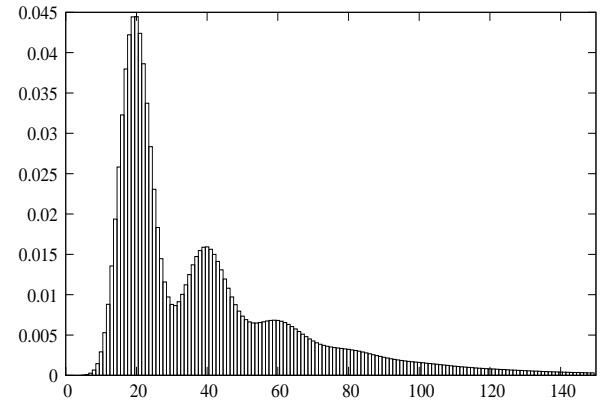

Figure 4: The PMF of a univariate GDGE distribution when $\alpha=1.5, \theta=0.01, p=e^{-0.1}$. 